\title{
INVESTIGACIONES
}

\section{Acceso, tiempo y propósito de uso de tecnologías digitales en estudiantes de pedagogía chilenos*}

\author{
Access, time and purposes of use of digital technologies \\ in Chilean teacher-training students
}

\author{
Cristian Cerda ${ }^{a}$,José L. Saiz, Lorena Villegas ${ }^{c}$, Miriam León ${ }^{d}$ \\ ${ }^{a}$ Departamento de Educación, Universidad de La Frontera \\ Correo electrónico: cristian.cerda@ufrontera.cl \\ ${ }^{\text {b }}$ Departamento de Psicología, Universidad de La Frontera \\ Correo electrónico: jose.saiz@ufrontera.cl \\ ${ }^{c}$ Departamento de Artes y Diseño, Universidad Católica de Temuco \\ Correo electrónico: 1villegas@uct.cl

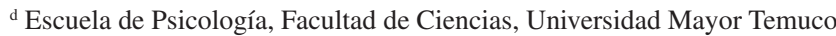 \\ Correo electrónico:m.leon05@ufromail.cl
}

\begin{abstract}
RESUMEN
Esta investigación tuvo por objetivo describir el acceso, tiempo y propósito de uso (académico, recreativo y social) de tecnologías digitales en estudiantes de pedagogía chilenos. Se examinó, además, si ser usuario académico se asocia con variables sociodemográficas, académicas y aprendizaje autodirigido. El análisis de las respuestas dadas por 546 participantes a cuestionarios reveló elevados niveles de acceso a dispositivos y recursos digitales. Mientras WhatsApp y Facebook concentran un mayor uso social, YouTube es priorizado en uso recreativo y el Buscador de Google en uso académico. Se constató un mayor número de usuarios académicos en las mujeres que en los hombres. Ser usuario académico de tecnologías digitales se relacionó, en cada sexo, con mayores niveles de aprendizaje autodirigido. Estos resultados apoyan la necesidad de preparar más y mejores usuarios académicos que aprovechen las ventajas ofrecidas por las tecnologías digitales, en función de una mejor formación inicial docente.
\end{abstract}

Palabras clave: uso de tecnología en educación, formación inicial docente, estudio independiente

\section{ABSTRACT}

This research intends to analyze access, time and purpose of use (academic, recreational and social) of digital technologies in Chilean teacher-training students. It was also examined, if being an academic user is associated with sociodemographic, academics and self-directed learning variables. The analysis of the answers provided by 546 participants in the questionnaires revealed high level of access to equipment and digital resources. Meanwhile WhatsApp and Facebook concentrate a higher social use, YouTube is prioritized in recreational activities, and Google search engine in academic use. It was found a higher number of woman in the category academic users. Being an academic user of digital technologies, in both sexes, is linked with higher levels of self-directed learning. These results support the need to prepare more and better academic users, able to take the advantages that digital technologies offer, in order to get a better initial teacher education.

Key words: technology use in Education, initial teacher training, independent study

Este estudio fue financiado por el Fondo Nacional de Desarrollo Científico y Tecnológico de Chile, Proyecto FONDECYT $\mathrm{N}^{\circ} 1191193$. 


\section{INTRODUCCIÓN}

Desde la implementación en Chile el año 1993 del Proyecto Enlaces (Red de Informática Educativa Escolar del Ministerio de Educación), la integración de tecnologías digitales con fines educativos ha sido tema de interés permanente en el ámbito de las políticas educativas (Centro de Educación y Tecnología, 2008). La red Enlaces generó un cambio sin precedentes en la educación chilena al centrar en el rol del profesor la integración curricular de tecnologías digitales (Hinostroza, Hepp \& Cox, 2009). Gracias a esta iniciativa, miles de docentes y estudiantes tuvieron la posibilidad de interactuar con computadores, utilizando sistemas de comunicación, software educativo o programas de productividad. La integración curricular de tecnologías digitales, en sus distintos niveles, representa un desafío permanente para aquellos docentes que buscan aprovechar académicamente las ventajas que estas herramientas ofrecen.

Puesto que existe un alto consenso en que los ciudadanos del futuro deberán estar alfabetizados computacionalmente, las tecnologías digitales han pasado a ser parte de la denominada nueva pedagogía (World Economic Forum, 2016). La integración curricular de tecnologías digitales se acopla a la necesidad del mundo empresarial de contar con ciudadanos preparados para enfrentar con éxito los requerimientos emergentes, en muchos casos desconocidos, que la sociedad de la información y comunicación demanda (World Bank, 2016). De igual manera, desde hace más de diez años, organizaciones como The Partnership for 21st Century Skills (2009) hacen llamados al mundo educativo para que los estudiantes logren utilizar de manera efectiva tecnologías digitales para investigar, organizar y evaluar la información disponible. Junto con este consenso, existe además claridad que los aprendices digitales no podrán aprovechar los potenciales beneficios que estas herramientas brindan, si no cuentan con habilidades básicas en lectura, escritura y navegación informática. No basta con asegurar el acceso a tecnologías digitales, brecha ya superada en muchos países (OECD, 2015); actualmente es necesario poner el foco de atención en las actividades que las personas hacen cuando usan estas herramientas.

En muchas oportunidades se asume de forma equívoca que los jóvenes, por el simple hecho de ser tales y usar con relativa fluidez las tecnologías digitales, pueden aprovechar académicamente estas herramientas. El uso de diversas etiquetas como nativos digitales (Prensky, 2001), aprendices digitales (Brown, 2000), y generación digital (Tapscott, 2009) han llevado a la opinión pública, y en especial a las comunidades educativas, a creer erróneamente que la relación entre jóvenes, tecnologías digitales y aprendizaje es fluida, permanente, efectiva y que evoluciona potenciándose en el tiempo (Lluna \& Pedreira, 2017). Esta percepción incluso ha ido más allá, generando cuestionamientos sobre si es necesario enseñar competencias tecnológicas a las nuevas generaciones de aprendices digitales $(\mathrm{Ng}$, 2012) o si, pese al acceso masivo, existen variados modos de inserción digital de los jóvenes en las tecnologías (Livingstone \& Helsper, 2016). Más allá de estas miradas, es necesario preguntarse si los jóvenes autónomamente hacen un uso efectivo de estas herramientas o si, por el contrario, se trata de una creencia errónea basada en los elevados niveles de autoeficacia computacional que los jóvenes suelen proyectar y que la sociedad acepta de manera acrítica (Rohatgi, Scherer \& Hatlevik, 2016).

En general, se acepta ampliamente que la actual penetración de tecnologías digitales personales (smartphones, tabletas ${ }^{1}$ y computadores portables) ha reconfigurado los

Pese a que en Chile se utiliza en concepto anglosajón tablet, la Real Academia Española lo denomina tableta. 
propósitos de uso que las personas, y en especial los jóvenes, les asignan a estas herramientas (Boyd, 2014; Ito et al., 2010; Ito et al., 2008). En esta perspectiva, durante los últimos quince años se han desarrollado varias taxonomías para clasificar las distintas formas en que las personas interactúan con estos recursos. Debido a que las tecnologías han penetrado diversas áreas, las taxonomías existentes son heterogéneas y evolucionan junto con el avance tecnológico. Por ejemplo, la taxonomía de Howard, Rainie \& Jones (2001) se focaliza en el perfil de los usuarios (cibernautas, utilitarios, experimentadores, recién llegados), mientras que la planteada por Ortega, Recio, y Román (2007) destaca el grado de adopción de los usuarios (rezagados, confundidos u opositores, usuarios avanzados, seguidores, y no usuarios de Internet). Selwyn, Gorard \& Furlong (2006) por otra parte, atienden al tiempo de uso (usuarios frecuentes, usuarios ocasionales y no usuarios). Tipologías más recientes se han centrado en los propósitos de uso, como es el caso de Holmes (2011), quien divide dichos propósitos en foco informativo, recreativo y usuarios básicos. Un caso similar es la tipología de Blank \& Groselj (2014), que clasifica estos propósitos en entretenimiento, comercio, búsqueda de información, uso social, email, blogs, uso en producción, uso de medios clásicos, trabajo, y vicio. Es posible identificar dos debilidades comunes a la mayoría de estas taxonomías que dificultan la comprensión de lo que realmente hacen las personas con estas herramientas: sus categorías buscan perfilar a usuarios genéricos y suelen combinar erróneamente distintas dimensiones de uso de estas tecnologías.

Blank \& Groselj (2014) plantean que existen tres dimensiones de uso de tecnologías digitales: cantidad, variedad y tipos de usos. La cantidad considera la frecuencia de tiempo empleada al usar estas herramientas de manera cotidiana, lo cual no necesariamente se relaciona con el tiempo que las personas llevan usando estas tecnologías. La cantidad de tiempo empleado (frecuencia de uso) se mide en horas y puede ser distinta entre usuarios. Por otra parte, la variedad de usos contabiliza el número de acciones que desarrolla un usuario durante el tiempo que interactúa con los recursos digitales. Veamos un ejemplo para diferenciar estas dos dimensiones. En una situación de alta cantidad, pero baja variedad de uso, una persona puede estar conectada todo el día a YouTube viendo videos; en una situación de baja cantidad, pero alta variedad de uso, otra persona puede, durante una hora, escuchar música en Spotify, revisar su email, conversar en Facebook vía chat con un amigo sobre una noticia que vio en Twitter, mientras realiza los últimos ajustes a una tarea que debe subir a la plataforma virtual de su universidad. Tipos de uso, la tercera dimensión, considera los propósitos de usos en los cuales se pueden clasificar las actividades que realiza una persona cuando usa tecnologías digitales. Estas actividades pueden tener, entre muchas otras, un carácter recreativo, económico, social, académico, de producción, etc. Otras taxonomías semejantes suelen no distinguir, y a veces combinar, estas tres dimensiones; tal es el caso, por ejemplo, de la clasificación planteada por Brandtzæg (2010) la cual incluye a no usuarios, usuarios esporádicos, con foco en debatir, centrados en entretención, socializadores, acechadores, instrumentales, y avanzados.

Aunque, en general las taxonomías proponen una gran variedad de propósito de usos, en contextos educativos universitarios tales propósitos parecen ser mucho más acotados. Desde nuestra perspectiva, la formación inicial docente permitiría identificar tres tipos distintivos de uso de tecnologías digitales: uso académico, recreativo, y social. El uso académico implica emplear estas herramientas para buscar información, comunicarse académicamente con otros, realizar trabajos y tareas digitales y, como medio de apoyo, 
comprender temas de interés educativo. El uso recreativo consiste en la utilización lúdica de las tecnologías, sea individualmente o en colaboración con otros. El uso social considera la comunicación y el tratamiento de temas generales de interés común entre los usuarios, a través de redes sociales. Creemos que los tres propósitos mencionados son influidos por el contexto académico y social en el cual los estudiantes están insertos, e impulsados por motivaciones o necesidades personales ligadas al perfil académico de los usuarios. Estos propósitos de usos pueden ser abordados en términos de tiempo y variedad, asumiendo en esta última dimensión que los jóvenes, cuando usan tecnologías digitales, tienden a realizar varias acciones a la vez.

En el contexto de formación inicial docente en Chile, el uso académico de tecnologías digitales convive con el uso recreativo y social de estas herramientas (Cerda, HueteNahuel, Molina-Sandoval, Ruminot-Martel \& Saiz, 2017; Flores-Lueg \& Roig, 2016). Una de las razones que podría explicar este fenómeno es la transición que han tenido las tecnologías digitales desde las salas de clases, mediadas por un docente, a los bolsillos y mochilas de miles de estudiantes, quienes diariamente deben decidir qué harán con ellas. Si bien los diferentes propósitos de uso de tecnologías digitales pueden visualizarse en los jóvenes como naturales y obvios, la aparición de nuevas aplicaciones en los smartphones, hacen que estos propósitos varíen en función de intereses emergentes, como ocurrió el año 2016 con el fenómeno Pokemon Go. Si bien el tema de los propósitos de uso solo ha sido abordado tangencialmente en estudiantes secundarios chilenos (Hinostroza, Matamala, Labbé, Claro \& Cabello, 2015), la evidencia en estudiantes chilenos de pedagogía es aún más escasa.

Dado el influjo que tienen las tecnologías digitales en la adquisición autónoma del conocimiento docente (Cerda, 2013a; 2013b), la carencia identificada en la literatura subraya la necesidad de precisar qué hace el estudiante de pedagogía mientras emplea tales tecnologías. Además, esta necesidad es potenciada por el rol modelador en el uso de tecnologías digitales que muy probablemente tendrán los profesores en formación con sus futuros estudiantes. A partir de los antecedentes antes descritos esta investigación tuvo como objetivo analizar el empleo de tecnologías digitales en estudiantes de pedagogía. En particular se buscó: (1) describir el acceso, tiempo y propósitos de uso de dispositivo computacional y recursos digitales; (2) analizar si ser o no usuario académico de tecnologías digitales dependen de variables sociodemográficas y académicas propias del perfil académico de los participantes; y (3) examinar diferencias entre usuarios académicos y no académicos en términos de edad y aprendizaje autodirigido

Este estudio forma parte de una línea de investigación que busca profundizar la comprensión del rol que cumplen las tecnologías digitales como recurso de apoyo a la construcción autónoma del conocimiento docente en estudiantes de pedagogía chilenos. Este proceso, que se ejecuta de manera complementaria a la educación formal, permite el desarrollo de competencias digitales que, bien encaminadas, podrían ser esenciales para apoyar el aprendizaje permanente de los futuros educadores. La relación entre tecnologías digitales, aprendizaje autodirigido y formación docente se ha posicionado internacionalmente como un eje relevante en el desarrollo profesional de los educadores (Bullock, 2013; Fisher, Higgins, y Loveless, 2006). Una mayor y mejor comprensión de este fenómeno puede contribuir a la generación de políticas públicas más precisas, que promuevan el uso explícito de tecnologías digitales en función del conocimiento docente. 


\section{MÉTODO}

\subsection{PARTICIPANTES}

En esta investigación participaron 546 estudiantes de pedagogía (291 hombres, $M=22.10$ años, $D E=3.50 ; 255$ mujeres, $M=21.60$ años, $D E=3.21$ ), todos estudiantes regulares al segundo semestre de 2017 en seis carreras de enseñanza media de una universidad pública chilena. La distribución de participantes por niveles fue: primero (141, 25.8\%), segundo $(131,24.0 \%)$, tercero $(84,15.4 \%)$, cuarto $(76,13.9 \%)$, quinto $(74,13.6 \%)$ y rezagados $(40,7.3 \%)$. Mediante un muestreo no probabilístico por conveniencia (Salkind, 1998) se obtuvo una muestra que cubrió al 56\% de la población de estudiantes de pedagogía de esta universidad.

\subsection{INSTRUMENTOS}

Se empleó un cuestionario generado por el equipo de investigación, en el cual los participantes debían indicar (1) si tenían o no acceso a cuatro dispositivos (computador de escritorio, computador portátil, Smartphone, y tableta), (2) el tiempo empleado en cada uno de ellos durante la semana laboral y fin de semana, y (3) la distribución porcentual del tiempo según propósitos de uso (académico, recreativo, y social). Los mismos datos fueron solicitados respecto al empleo de cuatro recursos digitales (Facebook, Buscador de Google, YouTube, y WhatsApp). Además, se recolectó información sociodemográfica y académica de los participantes.

Complementariamente, los participantes contestaron la Escala Corta de Aprendizaje Autodirigido desarrollada por Fisher y King (2010). Este instrumento compuesto por 29 ítems que están organizados en tres factores (autogestión, deseos de aprender, y autocontrol), es respondido según una escala de cinco niveles que van desde 1 (No me describe) a 5 (Me describe totalmente). La estructura factorial de esta escala ha sido examinada en Chile, aunque con un enfoque exploratorio, en estudiantes de medicina (Fasce, Pérez, Ortiz, Parra \& Matus, 2011) y estudiantes de pedagogía (Cerda \& Saiz, 2015). Con los datos del presente estudio, Cerda y Saiz, (2018), empleando un enfoque confirmatorio, verificaron la estructura trifactorial de esta escala, la cual presentó adecuados niveles de confiabilidad: autogestión (.846), deseos de aprender (.841), autocontrol (.772), y escala total (.911).

\subsection{PROCEDIMIENTO}

Los datos fueron tomados en formato de papel y lápiz durante el segundo semestre del año 2017, en el contexto de clases, previa autorización de los profesores responsable de las asignaturas. Los participantes leyeron y firmaron un consentimiento informado aprobado por el comité de ética de la universidad. Posteriormente, se distribuyeron los instrumentos y se explicó de manera detallada cómo completarlos. Los estudiantes faltantes fueron invitados a participar en las clases posteriores. La participación fue voluntaria, sin entrega de compensación alguna. Cada participante recibió, al final del año, información descriptiva de sus resultados, comparándolos con el promedio obtenido por los estudiantes de su carrera y por la totalidad de los estudiantes de pedagogía que participaron del estudio. 


\subsection{ANÁLISIS DE DATOS}

Primero, por cada dispositivo y recurso digital se desarrollaron análisis descriptivos de acceso (frecuencia y porcentajes), tiempo de uso semanal y fin de semana (promedio de horas), junto con la distribución porcentual del tiempo invertido según propósitos de uso. Segundo, se creó una variable denominada uso académico, con dos categorías (usuario académico versus no usuario académico). Los primeros fueron definidos operacionalmente como participantes que reportaron un uso académico igual o superior a $65 \%$ en, al menos, un dispositivo o recurso digital. El incumplimiento de este criterio definió la condición de no usuario académico.

Tercero, se empleó la prueba $\chi^{2}$ para examinar la relación del uso académico con las variables sexo, carrera, año de ingreso a la carrera, estar al día en la malla curricular y haber aprobado el examen de competencia TIC. Además, se examinaron diferencias entre ser o no usuario académico, dentro de cada sexo, en función de la edad y los puntajes de aprendizaje autodirigido (autogestión, deseos de aprender, autocontrol y escala total). Cuando se encontraron diferencias, se calculó el valor $d$ de Cohen (1988) para determinar el tamaño del efecto.

\section{RESULTADOS}

\subsection{DESCRIPCIÓN DE ACCESO, TIPO Y PROPÓSITOS DE USO}

La Tabla 1 muestra los resultados obtenidos sobre dispositivos computacionales en términos de acceso, tiempo de uso y propósito de usos en porcentajes.

Tabla 1. Acceso, tiempo y propósito de uso de dispositivos computacionales

\begin{tabular}{|c|c|c|c|c|c|c|}
\hline \multirow{2}{*}{ Dispositivos } & \multicolumn{2}{|c|}{ Acceso } & \multicolumn{2}{|c|}{ Tiempo (hrs) } & \multirow{2}{*}{$\begin{array}{l}\text { Propósito } \\
\text { de Uso }\end{array}$} & \multirow{2}{*}{$\begin{array}{c}\% \\
\text { de Uso }\end{array}$} \\
\hline & $n$ & $\%$ & L-V & S-D & & \\
\hline Computador de Escritorio & 110 & 20.1 & 2.24 & 2.32 & $\begin{array}{l}\text { Académico } \\
\text { Social } \\
\text { Recreativo }\end{array}$ & $\begin{array}{l}\mathbf{5 4 , 3} \\
16,6 \\
29,7\end{array}$ \\
\hline Computador Portable & 510 & 93.4 & 4.31 & 4.45 & $\begin{array}{l}\text { Académico } \\
\text { Social } \\
\text { Recreativo }\end{array}$ & $\begin{array}{l}\mathbf{5 0 . 6} \\
16.2 \\
30.6\end{array}$ \\
\hline Smartphone & 522 & 95.6 & 7.00 & 6.68 & $\begin{array}{l}\text { Académico } \\
\text { Social } \\
\text { Recreativo }\end{array}$ & $\begin{array}{l}20.8 \\
49.7 \\
29.6\end{array}$ \\
\hline Tableta & 80 & 14.7 & 1.79 & 1.70 & $\begin{array}{l}\text { Académico } \\
\text { Social } \\
\text { Recreativo }\end{array}$ & $\begin{array}{l}41.0 \\
16.5 \\
\mathbf{4 2 . 5}\end{array}$ \\
\hline
\end{tabular}

Nota. $\mathrm{L}-\mathrm{V}=$ lunes a viernes. $\mathrm{S}-\mathrm{D}=$ sábado y domingo. Porcentajes más elevados aparecen destacados en negrita. 
En relación a los dispositivos, los resultados muestran un mayor acceso a smartphone $(95.6 \%)$ y computador portable $(93.4 \%)$, equipamiento a los que también se les dedica un mayor tiempo de uso, no evidenciándose grandes diferencias entre los días de semana y fin de semana. El uso académico es mayor en el computador de escritorio (54.3\%) y computador portable (50.6\%), mientras que el smartphone tiene un mayor el uso social (49.7\%) y la tableta un uso recreativo (42.5\%). La Tabla 2 muestra el mismo análisis en relación a los recursos digitales.

Tabla 2. Acceso, tiempo y propósito de uso de recursos digitales

\begin{tabular}{|c|c|c|c|c|c|c|}
\hline \multirow{2}{*}{$\begin{array}{l}\text { Recursos } \\
\text { Digitales }\end{array}$} & \multicolumn{2}{|c|}{ Acceso } & \multicolumn{2}{|c|}{ Tiempo (hrs) } & \multirow{2}{*}{$\begin{array}{c}\text { Propósito } \\
\text { de Uso }\end{array}$} & \multirow{2}{*}{$\begin{array}{c}\% \\
\text { de Uso }\end{array}$} \\
\hline & $n$ & $\%$ & L-V & S-D & & \\
\hline Facebook & 519 & 95.1 & 3.59 & 3.79 & $\begin{array}{l}\text { Académico } \\
\text { Social } \\
\text { Recreativo }\end{array}$ & $\begin{array}{l}15.0 \\
\mathbf{5 4 . 6} \\
30.4\end{array}$ \\
\hline Buscador de Google & 532 & 97.4 & 2.90 & 2.72 & $\begin{array}{l}\text { Académico } \\
\text { Social } \\
\text { Recreativo }\end{array}$ & $\begin{array}{r}\mathbf{6 4 . 2} \\
8.5 \\
27.2\end{array}$ \\
\hline YouTube & 518 & 94.9 & 3.06 & 3.47 & $\begin{array}{l}\text { Académico } \\
\text { Social } \\
\text { Recreativo }\end{array}$ & $\begin{array}{r}20.6 \\
8.3 \\
71.1\end{array}$ \\
\hline WhatsApp & 521 & 95.4 & 5.34 & 5.10 & $\begin{array}{l}\text { Académico } \\
\text { Social } \\
\text { Recreativo }\end{array}$ & $\begin{array}{l}20.1 \\
\mathbf{6 5 . 8} \\
14.1\end{array}$ \\
\hline
\end{tabular}

Nota. $\mathrm{L}-\mathrm{V}=$ lunes a viernes. S-D = sábado y domingo. Porcentajes más elevados aparecen destacados en negrita.

En promedio, un $95.7 \%$ de los participantes reporta tener acceso a los recursos digitales, con tiempos de uso similares entre días de semana y fin de semana. El Buscador de Google concentra el mayor porcentaje de uso académico (64.2\%), YouTube tiene un uso netamente recreativo $(71.1 \%)$, mientras que los mayores porcentajes de uso social se concentran en WhatsApp (65.8\%) y Facebook (54.6\%).

\subsection{RELACION DE USO ACADÉMICO CON VARIABLES DISCRETAS}

De los participantes, $339(62.1 \%)$ resultaron clasificados como usuarios académicos y 207 (37.9\%) como no usuarios académicos (37.9\%). La Tabla 3 muestra la distribución de los participantes según uso académico, nivel (años de permanencia en el programa de formación docente) y sexo. 
Tabla 3. Distribución de participantes según uso académico, nivel y sexo

\begin{tabular}{|l|c|c|c|c|c|}
\hline & & \multicolumn{2}{|c|}{ UA } & \multicolumn{2}{c|}{ No UA } \\
\hline Nivel & Sexo & n & $\%$ & n & $\%$ \\
\hline Primero & $\mathrm{H}$ & 40 & 54.8 & 33 & 45.2 \\
\hline & $\mathrm{M}$ & 52 & 76.5 & 16 & 23.5 \\
\hline Segundo & $\mathrm{H}$ & 35 & 48.0 & 38 & 52.0 \\
\hline & $\mathrm{M}$ & 43 & 74.1 & 15 & 25.9 \\
\hline Tercero & $\mathrm{H}$ & 21 & 47.7 & 23 & 52.3 \\
\hline \multirow{2}{*}{ Cuarto } & $\mathrm{M}$ & 29 & 72.5 & 11 & 27.5 \\
\hline \multirow{2}{*}{} & $\mathrm{H}$ & 20 & 55.6 & 16 & 44.4 \\
\hline Quinto & $\mathrm{M}$ & 24 & 60.0 & 16 & 40.0 \\
\hline & $\mathrm{H}$ & 25 & 58.1 & 18 & 41.9 \\
\hline Rezagados & $\mathrm{H}$ & 24 & 77.4 & 7 & 22.6 \\
\hline & $\mathrm{M}$ & 16 & 88.9 & 2 & 11.1 \\
\hline Total & & 339 & 62.1 & 207 & 37.9 \\
\hline
\end{tabular}

Nota. $\mathrm{UA}=$ usuario académico. No UA = no usuario académico. Sexo. $\mathrm{H}=$ Hombres, $\mathrm{M}=$ Mujeres

Ser o no usuario académico apareció asociado a la variable sexo, $\chi^{2}(1)=27.528, p=.001$, con un valor $d=.46$ que indica un tamaño muy próximo a mediano. Los residuos estandarizados corregidos de la tabulación cruzada mostraron, solo en mujeres, una frecuencia significativamente mayor de usuarias académicas $(73.7 \%)$ junto a una frecuencia significativamente menor de no usuarias académicas $(26.3 \%) \mathrm{ps}<.01$. No se encontraron asociaciones significativas de uso académico con las variables carrera $\chi^{2}(5)=9.218, p=.101$; año de ingreso a la universidad $\chi^{2}(5)=2.441, p=.785$; estar al día en la malla curricular $\chi^{2}(1)$ $=2.996, p=.083$; ni haber aprobado la certificación de tecnologías digitales requerida a todos los estudiantes de la universidad $\chi^{2}(1)=1.566, p=.211$.

\subsection{RELACION DE USO ACADÉMICO CON VARIABLES CONTINUAS}

Los usuarios académicos incluyeron a 151 hombres y 188 mujeres, en tanto que los usuarios no académicos fueron 140 hombres y 67 mujeres. Mediante la prueba no paramétrica U de Mann-Whitney, usuarios académicos versus no usuarios académicos fueron comparados en términos de las variables continuas edad y factores de aprendizaje autodirigido ya que, previamente, la prueba de Kolmogorov-Smirnov mostró que ninguna de estas variables poseía una distribución normal $p \mathrm{~s}<.001$. 
Tabla 4. Comparación de usuarios y no usuarios académicos según edad y factores de aprendizaje autodirigido, por sexo.

\begin{tabular}{|l|c|c|c|c|c|}
\hline & UA & No UA & & & \\
\hline Variables & RM & RM & $z$ & $p$ & $\boldsymbol{d}$ \\
\hline Hombres & & & & & \\
\hline Edad & 146.1 & 145.9 & .028 & .978 & - \\
\hline Autogestión & 160.5 & 130.3 & 3.062 & .002 & .364 \\
\hline Deseos de Aprender & 162.8 & 128.0 & 3.536 & $<.001$ & .423 \\
\hline Autocontrol & 164.1 & 126.5 & 3.825 & $<.001$ & .459 \\
\hline Escala Total & 165.3 & 125.2 & 4.060 & $<.001$ & .490 \\
\hline Mujeres & & & & & \\
\hline Edad & 130.0 & 122.4 & .735 & .462 & - \\
\hline Autogestión & 137.1 & 102.4 & 3.316 & .001 & .424 \\
\hline Deseos de Aprender & 133.4 & 112.9 & 1.964 & .050 & .247 \\
\hline Autocontrol & 135.2 & 107.8 & 2.624 & .009 & .332 \\
\hline Escala Total & 136.5 & 104.1 & 3.095 & .002 & .395 \\
\hline
\end{tabular}

Nota. UA = usuario académico. No UA = no usuario académico. $\mathrm{RM}=$ rango medio. $\mathrm{z}=$ valor $\mathrm{z}$ de prueba $\mathrm{U}$ de Mann-Whitney.

Como se puede observar en la Tabla 4, no se obtuvieron diferencias significativas en cuanto a edad, en ninguno de los sexos. En cambio, en ambos sexos, los usuarios académicos obtuvieron puntuaciones significativamente mayores a los no usuarios académicos en los cuatro puntajes de aprendizaje autodirigido. El tamaño de estas diferencias fue entre pequeño y mediano (Cohen, 1988).

\section{DISCUSIÓN}

Esta investigación analizó el acceso, tiempo y propósitos de uso (académico, recreativo y social) de tecnologías digitales y, además, examinó la relación entre ser o no usuario académico con variables sociodemográficas y académicas y aprendizaje autodirigido, en estudiantes de pedagogía chilenos. Los resultados muestran un acceso mayoritario a smartphone y computador personal y un elevado acceso promedio a recursos digitales como Facebook, Buscador de Google, YouTube y WhatsApp. El uso académico es mayor a través del computador, sea de escritorio o portable, y empleando el recurso digital Buscador de Google. El ser o no usuario académico apareció asociado a la variable sexo y, dentro de cada sexo, a aprendizaje autodirigido. 
La revisión de la literatura identificó un conjunto de taxonomías que incluyen los propósitos de uso utilizados en este estudio. El uso recreativo puede ser observado en las taxonomías de Shah, Kwak \& Holbert (2001), Heim y Brandtzæg (2007), Brandtzæg (2010), Brandtzæg, Heim \& Karahasanović (2011) y Holmes (2011), mientras que el uso social se encuentra en la taxonomía de Johnson \& Kulpa (2007), Brandtzæg (2010) y Brandtzæg \& Heim (2010). Un patrón común en la mayoría de las taxonomías revisadas es que no consideran de manera explícita propósitos de uso académico. Esto podría ser explicado debido a que las taxonomías, en general, describen perfiles genéricos de usuarios, no acotados a contextos específicos. Pese a esto, es posible apreciar que algunas categorías podrían ser consideradas académicas, como búsqueda de información, generación de material digital y comunicación con otros. A diferencias de las taxonomías citadas, las desarrollas en contextos escolares por van Deursen \& van Dijk (2014) e Hinostroza et al. (2015), incluyen el uso académico de tecnologías digitales.

Por otra parte, los datos muestran una tendencia predecible en la relación entre dispositivos, recursos digitales y propósitos de usos. Tiene sentido que el mayor uso académico se efectúe a través del computador de escritorio o portable, que el uso social sea mayor en el smartphone y que la tableta tenga un mayor porcentaje de uso recreativo. Una tendencia similar ocurre con los recursos digitales, donde Facebook y WhatsApp concentran un mayor porcentaje de uso social, YouTube de uso recreativo y el Buscador de Google de uso académico. Un hecho no esperado y que llama la atención, es la distribución porcentual de algunos propósitos de uso de recursos digitales. Este es el caso de YouTube, recurso empleado por el $94.9 \%$ de los estudiantes, pero con un bajo uso académico (20.6\%). Esta plataforma digital, ampliamente difundida en el mundo de la educación, ofrece un elevado número de videos educativos, por lo que cuesta comprender este bajo porcentaje de uso académico. Además, si se piensa que la información que buscan los usuarios en YouTube corresponde a videos y no a música (en la actualidad gran parte de la demanda de música en se ha concentrado en plataformas como Spotify y Apple Music), el bajo porcentaje de uso académico podría ser explicado por varios factores: la inexistencia de recursos digitales adecuados para apoyar la formación inicial docente, la incapacidad de los estudiantes de encontrar los recursos disponibles, la imposibilidad de usar recursos digitales cuyo idioma sea el inglés, o la escasa orientación entregada para usar estos recursos por los académicos responsables de la formación inicial docente. Es importante considerar que YouTube, pese a ser un recurso de gran potencial educativo, no fue ideado con dicha finalidad, más allá que existan números reportes que avalen su utilidad para encontrar información que apoye el aprendizaje académico (Bhatia, 2018; Moghavvemi, Sulaiman, Jaafar \& Kasem, 2018).

De manera complementaria, el mayor porcentaje de uso académico asignado al Buscador de Google (64.2\%) señala que los estudiantes de pedagogía privilegian la búsqueda de este tipo de información por sobre la revisión de videos educativos en YouTube $(20.1 \%)$, la comunicación entre pares con fines académicos a través de WhatsApp $(20.1 \%)$ o Facebook (15.0\%). Los datos obtenidos en relación a la búsqueda de información son elevados y similares a los reportados en la prueba PISA 2011 en la cual un 66\% de los estudiantes chilenos de enseñanza media declaró realizar dicha actividad (OECD, 2010b). Este porcentaje de empleo de búsqueda de información también es similar al obtenido por Claro et al (2012), quienes, a través de la medición cuantitativa en 1185 jóvenes chilenos de 15 años, reportaron que mientras un $73 \%$ de los participantes es capaz de buscar información en la web. Pese a este relativamente alto porcentaje, estos mismos 
autores indican que un $51 \%$ de los jóvenes pudo organizar la información obtenida, un 45\% trasmitirla, un $36 \%$ evaluar su calidad, un $29 \%$ integrar ideas propias en ella, y solo un $17 \%$ refinar esa información creando un producto digital. Estos resultados son consistentes con los obtenidos por Saavedra \& Cerda (2013) quienes, a través de un abordaje cualitativo, centrado en observación no participante, analizaron patrones de búsqueda, análisis y usos de información digital en estudiantes universitarios. Los resultados mostraron que los estudiantes solo alcanzan la segunda fase del proceso recomendado por expertos, de búsqueda de información en la web; este proceso incluye: (i) definir el problema de investigación; (ii) buscar y evaluar información; (iii) analizar la información; (iv) sintetizar y utilizar la información. Estos hallazgos, más allá de ser obtenidos a través de aproximaciones metodológicas distintas, demandan reflexionar sobre la incapacidad de los estudiantes para realizar tareas de mayor complejidad en la búsqueda de información.

Por otra parte, los resultados de la relación entre uso académico de tecnologías digitales sexo son coherentes con estudios nacionales e internacionales. A nivel nacional, los datos del SIMCE TIC muestran que las mujeres realizan un mayor uso académico de tecnologías digitales que los hombres (Hinostroza et al., 2015). El análisis internacional, con datos de la prueba PISA, reporta que las mujeres, cuando usan tecnologías digitales en su tiempo libre, expresan una mayor tendencia a comunicarse y desarrollar actividades académicas, pese a tener, en general, menores niveles de autoeficacia computacional que los hombres. Estos últimos canalizan su mayor nivel de autoeficacia computacional hacia actividades recreativas o de programación (OECD, 2010b). Los resultados globales de PISA, contrastan con los obtenidos en la medición 2013 de la prueba ICILS (International Computer and Information Literacy Study), en donde las mujeres chilenas presentan un mayor nivel de autoeficacia computacional que sus pares masculinos (Fraillon, Ainley, Schulz, Friedman \& Gebhardt, 2014b), hecho que podría explicar la tendencia en mujeres de favorecer los usos comunicativos y académicos al emplear tecnologías digitales.

La asociación encontrada entre uso académico de tecnologías digitales y aprendizaje autodirigido es coherente con resultados obtenidos por Rashid y Asghar (2016) en estudiantes universitarios que revelan que, mientras mayor es el nivel de aprendizaje autodirigido, mayor tiende a ser el uso autónomo de tecnologías digitales. Complementariamente, llama la atención que, pese a que el uso académico es dependiente del sexo de los participantes (concentra un mayor número de mujeres), los usuarios académicos de ambos sexos presenten mayores niveles que sus pares no usuarios académicos, en los cuatro puntajes de aprendizaje autodirigido. Mediciones previas de aprendizaje autodirigido en esta población, sin considerar el uso de tecnologías digitales, también mostraron diferencias significativas a favor de las mujeres (Cerda \& Saiz, 2015; Parra, Cerda, López \& Saiz, 2014).

El hecho que los jóvenes tengan acceso y sean usuarios asiduos de tecnologías digitales no implica necesariamente que puedan utilizar eficazmente estas herramientas con propósitos académicos. La identificación de relaciones heterogéneas entre jóvenes y tecnologías digitales ya ha sido identificada en Chile por Sánchez, Salinas, Contreras \& Meyer (2011), quienes plantean que, aún cuando el acceso y tiempo de uso de las tecnologías digitales pueden ser similares entre los jóvenes, los propósitos de uso varían en función de la pertenencia a distintos grupos socioeconómicos. Esto permite aseverar que los integrantes de una misma generación no comparten un perfil único, contrariando así la afirmación, sostenida internacionalmente, sobre la existencia de una población homogénea de nativos digitales. Desde esta perspectiva, se confirmaría que los usuarios de tecnologías digitales 
en Chile están siendo afectados por una segunda brecha digital, que separa a aquellos jóvenes que aprovechan académicamente los beneficios de las tecnologías digitales versus los que no lo hacen (OECD, 2010a). Pedró (2011) señala que el nivel socioeconómico de los jóvenes actuaría como una barrera que impediría a los grupos más desposeídos acceder a los beneficios académicos que brindan las tecnologías digitales. La asociación entre nivel socioeconómico y propósitos de uso también ha sido constatada a través de la medición de la prueba ICILS (Agencia de Calidad de la Educación, 2015). Resultados similares obtuvieron también Claro et al. (2012) al medir habilidades avanzadas en el empleo de teconologías digitales. Por otra parte, existe claridad en la literatura que los distintos propósitos de usos de tecnologías digitales van ligados a los niveles de alfabetización computacional de los jóvenes (Fraillon, Ainley, Schulz, Friedman \& Gebhardt, 2014a).

Los resultados obtenidos en este estudio invitan a reflexionar sobre el nivel y rol que cumple el capital cultural de los estudiantes de pedagogía cuando interactúan con tecnologías digitales. El análisis de la prueba PISA 2016 mostró que mientras mayor es el capital cultural de los estudiantes, como debería ser el caso de los estudiantes universitarios, mayor tiende a ser el uso de tecnologías digitales con fines académicos (OECD, 2010a). El presente estudio corrobora esa relación al identificar una cantidad sustantiva de usuarios académicos $(62.1 \%)$ entre los estudiantes. La mayor concentración de mujeres $(73.7 \%)$ entre los usuarios académicos parece señalar que ellas estarían aprovechando de mejor manera el capital cultural que poseen, en función de los usos académicos de las tecnologías digitales.

Quizás, en lugar de hablar de capital cultural, sea necesario ajustar dicho análisis a la disposición y competencias digitales que las personas poseen. En esta perspectiva, pareciera más apropiado utilizar el concepto de capital digital. De acuerdo a Park (2017b), el capital digital puede ser entendido como el "conjunto predeterminado de disposiciones que influyen en la forma en cómo las personas se vinculan con las tecnologías digitales" (p. 27). Esto explicaría cómo un determinado grupo de personas (en este caso estudiantes de pedagogía) adoptan diversos propósitos de uso de tecnologías digitales. Park (2017a) plantea que existen diversos desafíos que inciden en la forma en que las tecnologías digitales son adoptadas, una vez logrado su acceso. El primer desafío obedece al hecho que las tecnologías digitales están en constante evolución, proveyendo de nuevas oportunidades, pero presentando a su vez, nuevos requerimientos, lo que demanda una permanente actualización de dispositivos y recursos, junto con las competencias digitales para emplearlos. Un segundo desafío deriva del hecho que las tecnologías digitales están determinadas por las normas sociales y culturales, lo que hace que la implementación de los diversos propósitos de usos sea un proceso permeable al contexto en el cual interactúan los usuarios. Finalmente, el tercer desafío, es lograr que los usuarios de estas herramientas tengan las competencias digitales necesarias para usarlas adecuadamente. Una mirada amplia de la inclusión digital ayudaría a entender cómo se generan nuevos usos de estas herramientas.

Futuras investigaciones podrían abordar este proceso desde ópticas cualitativas y cuantitativas. Al respecto, sería relevante indagar, desde una óptica cualitativa, los factores que llevan a los estudiantes de pedagogía a privilegiar un tipo de propósito de uso por sobre otro o examinar cómo los distintos propósitos de uso se complementan en función de la construcción de un perfil de capital digital. Además, se requiere indagar la existencia de otros propósitos de uso que puedan ser integrados a los utilizados en este estudio. Junto con esto, es necesario seguir generando análisis cuantitativos en los cuales se explore la asociación del uso académico con logro académico y variables no cognitivas 
como motivación, tenacidad académica, inteligencia fija y de crecimiento, involucramiento académico y hábitos de estudio. La identificación de nuevas asociaciones puede contribuir a develar posibles antecedentes y consecuencias del uso académico, contribuyendo a la generación de intervenciones educativas que potencien este uso particular de las tecnologías digitales.

Finalizando, es necesario indicar que, como toda investigación científica, este trabajo presenta algunas limitaciones. Primero, se espera que exista coherencia entre lo reportado por los estudiantes y lo que sucede en la realidad. Segundo, se asume que el acceso, tiempo y propósito medidos se superponen, debido a las prácticas multitarea propias de las generaciones actuales (Cardoso-Leite, Green \& Bavelier, 2015; Wallis, 2010). Tercero, delimitar previamente la medición de determinado dispositivos y recursos digitales podría inhibir la identificación de otros propósitos de uso que los estudiantes de pedagogía realizan. Finalmente, para entender mejor la situación del uso académico en comparación con los otros propósitos de uso, se hace necesario considerar que el empleo de estas de tecnologías está determinado por la disponibilidad, acceso y orientación académica que se les asignen. Un claro ejemplo de esto, es la actual política de las compañías telefónicas de brindar acceso gratuito a redes sociales y servicios de música en demanda para smartphones, lo que lleva a un mayor uso social y recreativo, en contraste con la inexistencia de acceso gratuito a recursos educativos.

\section{CONCLUSIÓN}

Se puede establecer que los propósitos de uso de tecnologías digitales no son exclusivos de un determinado dispositivo o computacional o recurso digital. Si bien los estudiantes de pedagogía encuestados privilegian un determinado propósito de uso por sobre otro, los tres propósitos mencionados pueden ser observados tanto en dispositivos como recursos digitales. Los usuarios académicos, de cada sexo, presentan un mayor nivel de aprendizaje autodirigido, sugiriendo que los estudiantes autodirigidos serían quienes obtienen el mayor provecho al utilizar académicamente estas herramientas. Futuros estudios en esta línea podrían contribuir a la identificación de áreas factibles de ser intervenidas, que reorienten la formación de competencias digitales en los estudiantes de pedagogía a fin de construir o afianzar un capital digital que promueva de forma explícita un mayor y mejor uso académico de las tecnologías digitales disponibles, en función de una mayor y mejor adquisición de conocimiento docente.

\section{REFERENCIAS BIBLIOGRÁFICAS}

Agencia de Calidad de la Educación. (2015). ICILS 2013: El desafío de cerrar la brecha digital en los jóvenes chilenos. Santiago, Chile: Agencia de Calidad de la Educación. Ministerio de Educación.

Bhatia, A. (2018). Interdiscursive performance in digital professions: The case of YouTube tutorials. Journal of Pragmatics, 124, 106-120. doi:10.1016/j.pragma.2017.11.001

Blank, G., \& Groselj, D. (2014). Dimensions of Internet use: Amount, variety, and types. Information, Communication \& Society, 17(4), 417-435. doi:10.1080/1369118X.2014.889189

Boyd, D. (2014). It's complicated: The social lives of networked teens. New Haven: Yale University 
Press.

Brandtzæg, P. B. (2010). Towards a unified Media-User Typology (MUT): A meta-analysis and review of the research literature on media-user typologies. Computers in Human Behavior, 26(5), 940-956. doi:10.1016/j.chb.2010.02.008

Brandtzæg, P. B., \& Heim, J. (2010). A typology of social networking sites users. International Journal of Web Based Communities, 7(1), 28-51.

Brandtzæg, P. B., Heim, J., \& Karahasanović, A. (2011). Understanding the new digital divide: A typology of Internet users in Europe. International Journal of Human-Computer Studies, 69(3), 123-138. doi:10.1016/j.ijhcs.2010.11.004

Brown, J. S. (2000). Growing up: Digital: How the web changes work, education, and the ways people learn. Change: The Magazine ofHigherLearning, 32(2), 11-20. doi:10.1080/00091380009601719

Bullock, S. M. (2013). Using digital technologies to support self-directed learning for preservice teacher education. Curriculum Journal, 24(1), 103-120. doi:10.1080/09585176.2012.744695

Cardoso-Leite, P., Green, C. S., \& Bavelier, D. (2015). On the impact of new technologies on multitasking. Developmental Review, 35, 98-112. doi:10.1016/j.dr.2014.12.001

Centro de Educación y Tecnología. (2008). Enlaces: 15 años integrando tecnologías a la educación chilena. Santiago, Chile: Centro de Educación y Tecnología. Ministerio de Educación.

Cerda, C. (2013a). Desarrollo profesional docente autodirigido y tecnologías de la información y comunicación. Revista Ibero-Americana de Estudos em Educação, 8(1), 241-251.

Cerda, C. (2013b). Formación continua docente y tecnologías digitales. En S. Fridman \& R. EdelNavarro (Eds.), Ciencias, tecnologías y culturas: Educación y nuevas tecnologías (pp. 425-432). México, DF: RILET.

Cerda, C., Huete-Nahuel, J., Molina-Sandoval, D., Ruminot-Martel, E., \& Saiz, J. L. (2017). Uso de tecnologías digitales y logro académico en estudiantes de pedagogía chilenos. Revista Estudios Pedagógicos., 43(3), 119-133.

Cerda, C., \& Saiz, J. L. (2015). Aprendizaje autodirigido en estudiantes de pedagogía chilenos: Un análisis psicométrico. Suma Psicológica, 22(2), 129-136. doi:10.1016/j.sumpsi.2015.08.004

Cerda, C., \& Saiz, J. L. (2018). Análisis psicométrico de la Escala Corta de Aprendizaje Autodirigido de Fisher en estudiantes de pedagogía chilenos. Manuscrito en preparación.

Claro, M., Preiss, D. D., San Martín, E., Jara, I., Hinostroza, J. E., Valenzuela, S., . . Nussbaum, M. (2012). Assessment of 21st century ICT skills in Chile: Test design and results from high school level students. Computers \& Education, 59(3), 1042-1053. doi:10.1016/j.compedu.2012.04.004

Cohen, J. (1988). Statistical power analysis for the behavioral sciences (2nd ed. ed.). Hillsdale, NJ: Erlbaum.

Fasce, E., Pérez, C., Ortiz, L., Parra, P., \& Matus, O. (2011). Estructura factorial y confiabilidad de la Escala de Aprendizaje Autodirigido de Fisher, King \& Tague en alumnos de medicina chilenos. Revista Médica de Chile, 139, 1428-1434.

Fisher, M. J., \& King, J. (2010). The Self-directed Learning Readiness Scale for Nursing Education revisited: A confirmatory factor analysis. Nurse Education Today, 30(1), 44-48. doi:10.1016/j. nedt.2009.05.020

Fisher, T., Higgins, C., \& Loveless, A. (2006). Teachers learning with digital technologies: A review of research and projects. Bristol, UK: Futurelab.

Flores-Lueg, C., \& Roig, R. (2016). Percepción de estudiantes de pedagogía sobre el desarrollo de su competencia digital a lo largo de su proceso formativo. Revista Estudios Pedagógicos., 42(3), 129-148. doi:10.4067/S0718-07052016000400007

Fraillon, J., Ainley, J., Schulz, W., Friedman, T., \& Gebhardt, E. (2014a). Preparing for life in a digital age: The IEA International Computer and Information Literacy Study International report. New York, NY: Springer Berlin Heidelberg.

Fraillon, J., Ainley, J., Schulz, W., Friedman, T., \& Gebhardt, E. (2014b). Students' use of and engagement with ICT at home and school. En J. Fraillon, J. Ainley, W. Schulz, T. Friedman, 
\& E. Gebhardt (Eds.), Preparing for life in a digital age: The IEA International Computer and Information Literacy Study International Report (pp. 125-166). Cham: Springer International Publishing.

Heim, J., \& Brandtzæg, P. B. (Diciembre, 2007). Patterns of media usage and the non-professional users. En D. Geerts, P.B. Brandtzæg, M. Obrist, M. Tscheligi (Presidencia), Supporting nonprofessional users in the new media landscape. Simposio llevado a cabo en la conferencia de CHI '07, the SIGCHI Conference on Human Factors in Computing Systems, San Jose, CA.

Hinostroza, J. E., Hepp, P., \& Cox, C. (2009). National policies and practices on ICT in education: Chile. En T. Plomp, R. E. Anderson, N. Law, \& A. Quale (Eds.), Cross-national information and communication technology: Policies and practices in education. Charlotte, North Carolina: Information Age Publishing.

Hinostroza, J. E., Matamala, C., Labbé, C., Claro, M., \& Cabello, T. (2015). Factors (not) affecting what students do with computers and internet at home. Learning, Media and Technology, 40(1), 43-63. doi:10.1080/17439884.2014.883407

Holmes, J. (2011). Cyberkids or divided generations? Characterising young people's internet use in the UK with generic, continuum or typological models. New Media \& Society, 13(7), 11041122. doi: $10.1177 / 1461444810397649$

Howard, P., Rainie, L., \& Jones, S. (2001). Days and nights on the Internet: The Impact of a diffusing technology. American Behavioral Scientist, 45(3), 383-404. doi:10.1177/0002764201045003003

Ito, M., Antin, J., Finn, M., Law, A., Manion, A., Mitnick, S., . . Horst, H. A. (2010). Hanging out, messing around, and geeking out: Kids living and learning with new media. Cambridge, Mass.: MIT Press.

Ito, M., Horst, H., Bittanti, M., Boyd, D., Herr-Stephenson, B., Lan, P. G., . . Robinson, L. (2008). Living and learning with new media: Summary of findings from the Digital Youth Project. Chicago, IL: The MacArthur Foundation.

Johnson, G. M., \& Kulpa, A. (2007). Dimensions of online behavior: Toward a user typology. CyberPsychology \& Behavior, 10(6), 773-780. doi:10.1089/cpb.2007.0043

Livingstone, S., \& Helsper, E. (2016). Gradations in digital inclusion: Children, young people and the digital divide. New Media \& Society, 9(4), 671-696. doi:10.1177/1461444807080335

Lluna, S., \& Pedreira, J. (2017). Los nativos digitales no existen: Cómo educar a tus hijos para un mundo digital. Barcelona: Deusto.

Moghavvemi, S., Sulaiman, A., Jaafar, N. I., \& Kasem, N. (2018). Social media as a complementary learning tool for teaching and learning: The case of youtube. The International Journal of Management Education, 16(1), 37-42. doi:10.1016/j.ijme.2017.12.001

$\mathrm{Ng}, \mathrm{W}$. (2012). Can we teach digital natives digital literacy? Computers \& Education, 59(3), 10651078. doi:10.1016/j.compedu.2012.04.016

OECD. (2010a). Are the new millennium learners making the grade? Technology use and educational performance in PISA. Paris, France: OECD Publishing.

OECD. (2010b). Students' use of information and communication technologies and the role of confidence. En OECD (Ed.), Are the new millennium learners making the grade? Technology use and educational performance in PISA (pp. 65-120). Paris, France: Autor.

OECD. (2015). Students, computers and learning. making the connection. Paris: OECD.

Ortega, J., Recio, M., \& Román, M. (2007). Diffusion and usage patterns of Internet services in the European Union. Information Research, 12(2). Recuperado el 22 de enero de 2018 desde http:// www.informationr.net/ir/12-2/paper302.html

Park, S. (2017a). Introduction. En S. Park (Ed.), Digital Capital (pp. 1-10). London: Palgrave Macmillan UK.

Park, S. (2017b). Understanding digital capital within a user's digital technology ecosystem. En S. Park (Ed.), Digital Capital (pp. 63-82). London: Palgrave Macmillan UK.

Parra, J., Cerda, C., López, Ó., \& Saiz, J. L. (2014). Género, autodirección del aprendizaje y 
desempeño académico en estudiantes de pedagogía. Educación y Educadores, 17(1), 91-107. doi:10.5294/edu.2014.17.1.5

Pedró, F. (2011). Tecnología y escuela: Lo que funciona y por qué. Madrid, España: Fundación Santillana.

Prensky, M. (2001). Digital Natives, Digital immigrants, Part 1. On the Horizon, 9(5), 1-6. doi:10.1108/10748120110424816

Rashid, T., \& Asghar, H. M. (2016). Technology use, self-directed learning, student engagement and academic performance: Examining the interrelations. Computers in Human Behavior, 63, 604612. doi:10.1016/j.chb.2016.05.084

Rohatgi, A., Scherer, R., \& Hatlevik, O. E. (2016). The role of ICT self-efficacy for students' ICT use and their achievement in a computer and information literacy test. Computers \& Education, 102 (Supplement C), 103-116. doi:10.1016/j.compedu.2016.08.001

Saavedra, P., \& Cerda, C. (2013). Usos implícitos de estrategias de manejo de información en Internet. En S. Fridman \& R. Edel-Navarro (Eds.), Ciencias, tecnologías y culturas: Educación y nuevas tecnologías (pp. 393-398). México, DF: RILET.

Salkind, N. J. (1998). Métodos de investigación (3a ed.). México: Prentice Hall.

Sánchez, J., Salinas, A., Contreras, D., \& Meyer, E. (2011). Does the new digital generation of learners exist? A qualitative study. British Journal of Educational Technology, 42(4), 543-556. doi:10.1111/j.1467-8535.2010.01069.x

Selwyn, N., Gorard, S., \& Furlong, J. (2006). Adult learning in the digital age: Information technology and the learning society. London; New York: Routledge.

Shah, D. V., Kwak, N., \& Holbert, R. L. (2001). "Connecting" and "disconnecting" with civic life: Patterns of Internet use and the production of social capital. Political Communication, 18(2), 141-162. doi:10.1080/105846001750322952

Tapscott, D. (2009). Grown up digital: How the net generation is changing your world. New York: McGraw-Hill.

The Partnership for 21st Century Skills. (2009). Framework for 21st Century learning. Tucson, AZ: The Partnership for 21st Century Skills.

van Deursen, A. J., \& van Dijk, J. A. (2014). The digital divide shifts to differences in usage. New Media \& Society, 16(3), 507-526. doi:10.1177/1461444813487959

Wallis, C. (2010). The impacts of media multitasking on children's learning \& development: Report from a research seminar. New York, NY: The Joan Ganz Cooney Center at Sesame Workshop.

World Bank. (2016). World development report 2016: Digital dividends. Washington, DC: World Bank.

World Economic Forum. (2016). New vision for education: Fosterings and emotional learning through technology. The Boston Consulting Group. Cologny/Geneva: World Economic Forum. 